\title{
Monoclonal antibody-mediated neutralization of SARS-CoV-2 in an IRF9-deficient child
}

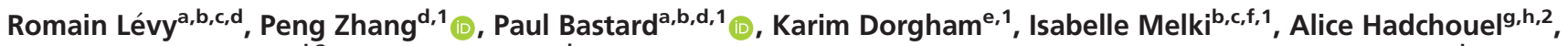 \\ George C. Hartoularos ${ }^{\mathrm{i}, 2}$, Bénédicte Neven ${ }^{\mathrm{b}, \mathrm{c}}$, Martin Castelle ${ }^{\mathrm{c}}$, Charlotte Roy ${ }^{\mathrm{g}}$, Tom Toin $^{\mathrm{g}}$, Laureline Berteloot ${ }^{\mathrm{j}}{ }^{\circ}$, \\ Lucy Bizien ${ }^{\mathrm{a}, \mathrm{b}}$, Hanène Abid ${ }^{\mathrm{k}}$, Marianne Burgardk, Nadhira Houhou-Fidouh', Flore Rozenberg ${ }^{\mathrm{m}}$,

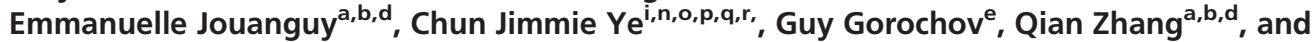 \\ Jean-Laurent Casanova ${ }^{\mathrm{a}, \mathrm{b}, \mathrm{d}, \mathrm{s}, 3}$ (1)
}

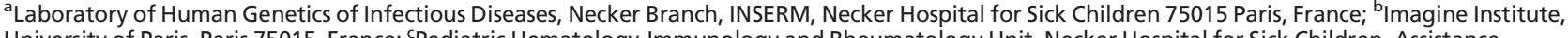
University of Paris, Paris 75015, France; ' $P$ Pediatric Hematology-Immunology and Rheumatology Unit, Necker Hospital for Sick Children, Assistance

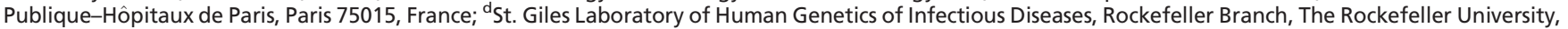
New York, NY 10065; ' Sorbonne Université, INSERM, Centre for Immunology and Microbial Infections-Paris, Pitié-Salpêtrière Hospital, Assistance

Publique-Hôpitaux de Paris, Paris 75013, France; ${ }^{f}$ General Pediatrics, Infectious Disease and Internal Medicine Department, Robert Debré Hospital, Assistance Publique-Hôpitaux de Paris, Paris 75019, France; '9Pediatric Pulmonary Department, Necker Hospital for Sick Children, Assistance Publique-Hôpitaux de Paris,

Paris 75015, France; ${ }^{h}$ Institut Necker-Enfants Malades, INSERM U1151, Paris 75015, France; 'Institute for Human Genetics, University of California, San Francisco, CA 94143; 'ंPediatric Radiology, Necker Hospital for Sick Children, Assistance Publique-Hôpitaux de Paris, Paris 75015, France; ${ }^{k}$ Department of Virology, Necker Hospital for Sick Children, Assistance Publique-Hôpitaux de Paris, Paris 75015, France; 'Department of Virology, INSERM, Infection, Antimicrobiens,

Modélisation, Evolution, UMR 1137, Bichat-Claude Bernard Hospital, University of Paris, Assistance Publique-Hôpitaux de Paris, Paris F-75018, France;

mDepartment of Virology, Cochin Hospital, University of Paris, Assistance Publique-Hôpitaux de Paris, Paris 75014, France; ${ }^{\mathrm{n}}$ Institute for Human Genetics,

University of California, San Francisco, CA 94143; ${ }^{\circ}$ Departments of Epidemiology and Biostatistics and Bioengineering and Therapeutic Sciences, University of

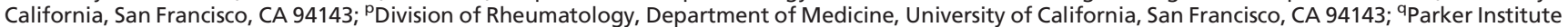

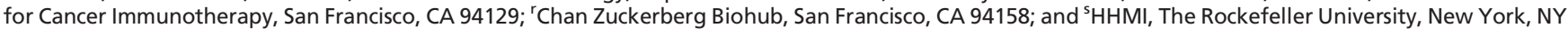
10065
\end{abstract}

Contributed by Jean-Laurent Casanova, September 27, 2021 (sent for review August 4, 2021; reviewed by Fabio Candotti and Caroline A. Jefferies)

We describe an unvaccinated child at risk for life-threatening COVID-19 due to an inherited deficiency of IRF9, which governs ISGF-3-dependent responses to type I and III interferons (IFN). She was admitted, with a high nasal SARS-CoV-2 load on day 1 of upper respiratory tract infection. She was viremic on day 2 and received casirivimab and imdevimab. Her clinical manifestations and viremia disappeared on days 3 and 4, respectively. Circulating SARS-CoV-2 virus induced the expression of IFN-stimulated genes in leukocytes on day 1, whereas the secretion of blood type I IFNs, which peaked on day 4, did not. Antibody-mediated SARS-CoV-2 neutralization is, therefore, sufficient to overcome a deficiency of antiviral IFNs.

COVID-19 | SARS-CoV-2 | inherited primary immunodeficiency | interferon | RNA-seq

nterindividual clinical variability in the course of SARSCoV-2 infection is huge, ranging from silent infection to fatal COVID-19 (1). We have reported that life-threatening COVID-19 pneumonia can be caused in about $20 \%$ of cases by rare inborn errors of Toll-like receptor (TLR)3-, TLR7-, or IRF7-dependent type I interferon (IFN) immunity $(2,3)$, or by the presence of autoantibodies (auto-Abs) neutralizing IFN- $\alpha 2$ or IFN- $\omega$, or more rarely IFN- $\beta$ (4-16). These auto-Abs were long known to exist but were previously thought to be clinically silent. In contrast, recessive and dominant inborn errors of the type I IFN-inducing and response pathways had previously been reported in patients with other severe viral illnesses, including severe influenza pneumonia (17-20). Surprisingly, we found within the population of patients with critical COVID-19 pneumonia and inborn errors of type I IFN immunity, four unrelated and previously healthy adults, aged 25 to $50 \mathrm{y}$, with autosomal recessive (AR) IRF7 $(n=2)$ or IFNAR1 $(n=2)$ deficiency. The absence of IRF7 prevents the amplification of type I and III IFNs, whereas that of IFNAR1 prevents cellular responses to type I IFNs (and their ensuing amplification). These findings revealed that patients with inborn errors of type I IFN immunity, particularly those with recessive deficiencies of IRF7 or IFNAR1, whose biochemical defects are complete, are at high risk of life-threatening COVID-19 pneumonia (21). They further suggested that the early administration of IFN- $\alpha$ or $-\beta$ in the course of SARS-CoV-2 infection might benefit patients with inborn errors impairing the production of type I IFNs (22), whereas IFN- $\beta$ administration may be beneficial in patients with neutralizing auto-Abs against IFN- $\alpha$ but not IFN- $\beta$ (23, 24). Moreover, these findings suggested that patients with inborn errors of the type I IFN response pathway or with auto-Abs neutralizing both IFN- $\alpha$ and IFN- $\beta$ should be

\section{Significance}

Life-threatening COVID-19 pneumonia can be caused by rare inborn errors of type I interferon (IFN) immunity, or by autoantibodies neutralizing IFN- $\alpha 2$ or IFN- $\omega$. In 2018 , we reported a girl with critical influenza pneumonia due to inherited IRF9 deficiency, a component of the ISGF-3 transcription factor. We report the course of COVID-19 in the same patient. She was admitted on day 1 of upper respiratory tract infection with viremia. Administration of SARS-CoV-2-specific neutralizing monoclonal antibodies on day 2 prevented the development of pneumonia. SARS-CoV-2-specific monoclonal antibodies were sufficient to overcome a lack of ISGF-3- and IRF9-dependent type I and type III IFN immunity to the virus. They should be considered in selected children at high risk of life-threatening COVID-19.

Author contributions: R.L., P.Z., P.B., Q.Z., and J.-L.C. designed research; R.L., P.Z., P.B., K.D., L. Bizien., N.H.-F., F.R., E.J., G.G., and Q.Z. performed research; P.Z., G.C.H., and C.J.Y. contributed new reagents/analytic tools; I.M., A.H., B.N., M.C., C.R., T.T., L. Berteloot., H.A., and M.B. contributed patient samples and clinical data; L. Berteloot., H.A., and M.B. contributed clinical data; R.L., P.Z., P.B., K.D., G.G., Q.Z., and J.-L.C. analyzed data; and R.L., P.Z., P.B., Q.Z., and J.-L.C. wrote the paper.

Reviewers: F.C., University Hospital of Lausanne; and C.A.J., Cedars-Sinai Health System.

The authors declare no competing interest.

This open access article is distributed under Creative Commons Attribution License 4.0 (CC BY).

${ }^{1}$ P.Z., P.B., K.D., and I.M. contributed equally to this work

${ }^{2}$ A.H. and G.C.H. contributed equally to this work.

${ }^{3}$ To whom correspondence may be addressed. Email: casanova@rockefeller.edu.

This article contains supporting information online at http://www.pnas.org/lookup/ suppl/doi:10.1073/pnas.2114390118/-/DCSupplemental.

Published October 26, 2021. 
treated differently, perhaps with monoclonal antibodies (mAbs) against SARS-CoV-2 (25-27).

In 2018, we reported AR complete IRF9 deficiency in a 5-yold French girl of Algerian ancestry (28). At the age of $2 \mathrm{y}$, she was admitted to an intensive care unit for severe influenza pneumonia due to influenza A virus (IAV). She required mechanical ventilation for $6 \mathrm{~d}$ for acute respiratory distress syndrome (ARDS) (28). She has since received prophylactic intravenous $\mathrm{IgG}$ every $3 \mathrm{wk}$ and has been vaccinated annually against influenza, which has considerably improved her clinical status, as she has developed no other severe viral illness. IRF9 is a crucial component of the type I and III IFN signaling pathways, as it associates with STAT1 and STAT2 to form the trimeric ISGF-3 transcription factor, which governs cellular antiviral responses to type I and III IFNs (29-31). We showed that the c. $991 \mathrm{G}>$ A mutant IRF9 allele in the patient was lossof-function, resulting in a lack of both ISGF-3 activation and ISGF-3-dependent IFN stimulated gene (ISG) induction following the stimulation of the patient's cells with IFN- $\alpha 2$ (28). Accordingly, the patient's fibroblasts displayed a high degree of susceptibility to IAV infection, which was not rescued by pretreatment with IFN- $\alpha 2$. Antiviral immunity was broadly impaired in vitro, as the patient's cells also displayed impaired cell-intrinsic immunity to parainfluenza virus and respiratory syncytial virus. In the context of our discovery of inborn errors of type I IFN underlying critical COVID-19 pneumonia (2-4, 21 ), these findings strongly suggested that this patient was at high risk of life-threatening COVID-19 pneumonia. The lack of ISGF-3 activation in response to both type I and type III IFNs, an immunological phenotype even more severe than that of patients with AR IRF7 deficiency, whose cells produce IFN- $\beta$ but do not amplify either type I or type III IFNs (19), further suggested that this patient might even develop a fulminant form of COVID-19 $(19,28)$. However, the parents refused to have their 8-y-old daughter vaccinated against SARS-CoV-2.

She was admitted to our unit on the first day of clinical manifestations consistent with mild, upper respiratory tract COVID19, including rhinitis, cough, fatigue, and low-grade fever $\left(38.2^{\circ} \mathrm{C}\right)$. On day 1 , PCR on a nasal swab revealed a very high load of SARS-CoV-2 (Ct: 16.5; $8.4 \log _{10}$ copies per milliliter) and showed the patient to be infected with the N501Y $\alpha$-variant (previously known as B.1.1.7) (32). SARS-CoV-2 was undetectable in the blood on day 1 , but viremia was detected on day 2 (Fig. 1A). Oxygen saturation was $97 \%$, respiratory rate was 20 breaths per minute, and physical examination found diminished breath sounds in the upper right lobe, consistent with previous examinations. Complete blood count was normal and blood C-reactive protein concentration was $7 \mathrm{mg} / \mathrm{L}$. A computed tomography (CT)-scan of the lungs on day 1 revealed bilateral curvilinear subpleural opacities, and excluded a moderate (i.e., nonhypoxemic) form of COVID-19 pneumonia, as all lesions were already present on a CT-scan at 5 years of age, consistent with sequelae of the patient's prior influenza ARDS (Fig. $1 B$ ).

On day 2, the patient received a single half-dose $(600 \mathrm{mg})$ of intravenous casirivimab and imdevimab, a combination of human $\mathrm{IgG1}$ neutralizing the receptor-binding domain of the SARS-CoV-2 spike protein $(25,33)$. Although there are
A

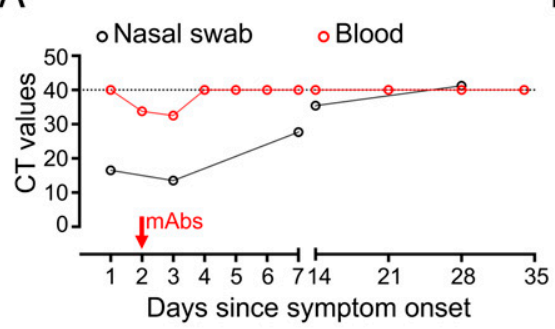

$\mathrm{C}$
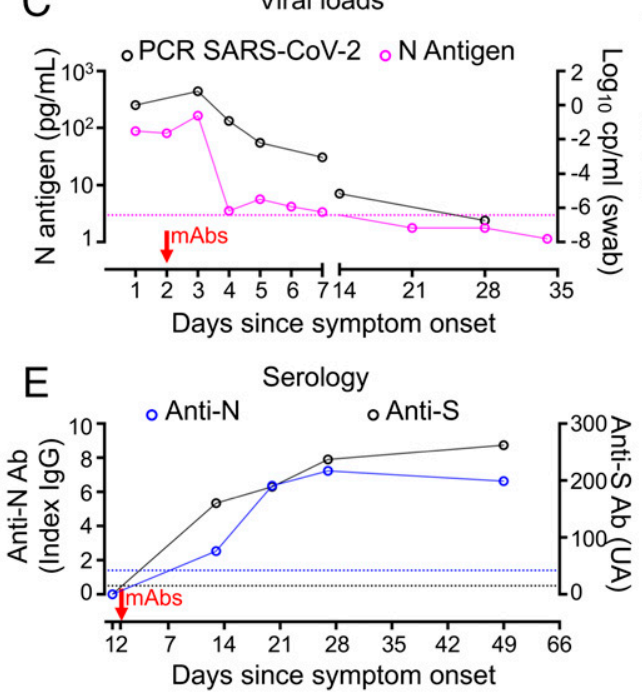

B

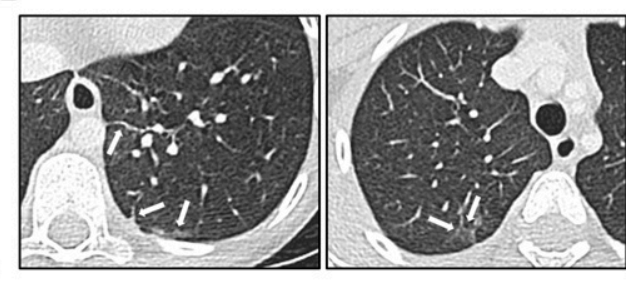

D IFN- $\alpha 2$

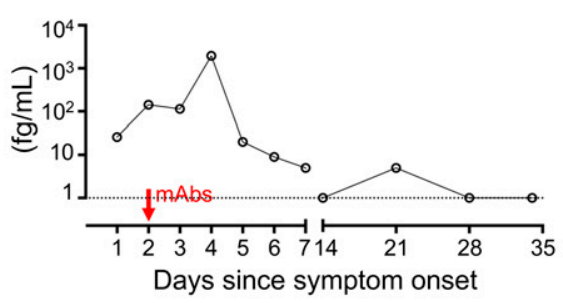

Fig. 1. (A) RT-PCR for SARS-CoV-2 on nasal swabs and blood on the day of symptom onset (day 1), and at various time points following treatment with casirivimab and imdevimab (mAbs), prescribed on day 2 of symptoms. $(B)$ CT of the lungs of the patient on day 1 , showing bilateral curvilinear subpleural opacities. (C) Decrease in viral load, in $\log _{10}$ copies per milliliter, in nasal swabs and decrease in $\mathrm{N}$-antigenemia (in pg/mL) following treatment with mAbs. (D) IFN- $\alpha 2$ determination by Simoa, on plasma collected from the patient on day 1 , and at various time points after mAb treatment. (E) Serological results for SARS-CoV-2 anti-nucleocapsid protein $(\mathrm{N})$ and spike protein $(\mathrm{S})$ antibodies in the patient's serum at various time points after the initiation of mAb treatment. 
currently no recommendations for administration of casirivimab and imdevimab in children (26), the strong rationale for this treatment was: 1) its recommended use in patients at high risk of critical COVID-19 pneumonia but not yet requiring oxygenotherapy $(25,34)$; 2) its efficacy in patients with high nasopharyngeal viral loads, as in this patient $(25) ; 3$ ) the known vulnerability of this IRF9-deficient patient and her cells to various respiratory viruses $(28)$; 4) the pulmonary sequelae of influenza ARDS in this patient $(28)$; 5) the occurrence in this patient of SARSCoV-2 viremia, which is associated with a high risk of respiratory deterioration and death $(35) ; 6)$ the high risk of hypoxemic COVID-19 pneumonia in patients with AR IFNAR1 or IRF7 deficiency $(2,19)$; and 7$)$ the lack of a better therapeutic option, as therapy with IFN- $\alpha$ or $-\beta$ was predicted to fail in the absence of ISGF-3 (as would treatment with IFN- $\lambda$, had it been available).

The patient did not receive any other medication, her symptoms and signs disappeared on day 3 , and she was discharged on day 8 , after a second CT-scan found no new pulmonary lesions. The most recent follow-up evaluation on day 50 was unremarkable; the patient was completely asymptomatic. Regular monitoring, by viral PCR on nasal swabs and blood, showed that SARS-CoV-2 viremia resolved on day 4 (Fig. $1 A$ ), whereas nasal viral load decreased strongly on day $7\left(-3.06 \log _{10}\right.$ copies per milliliter; Ct: $27.7 ; 5.3 \log _{10}$ copies per milliliter) (Fig. $1 A$ and $C$ ), reaching the threshold of detection on day 14 (Ct: 35.4 ; $3.2 \log _{10}$ copies per milliliter). SARS-CoV-2 antigenemia peaked on day 3 , with antigens undetectable from day 4 onward (Fig. $1 C$ ). The production of IFN- $\alpha 2$, as assessed by Simoa on plasma from the patient, was detectable on day $1(26 \mathrm{fg} / \mathrm{mL})$ and within the range reported in patients with COVID-19 not requiring mechanical ventilatory support $(10$ to $70,930 \mathrm{fg} / \mathrm{mL}$; median 3,240 fg/mL) (36). It increased until day $4(1,951 \mathrm{fg} /$ $\mathrm{mL})$, and then sharply decreased on day $5(20 \mathrm{fg} / \mathrm{mL})$, becoming undetectable by day 14 (Fig. $1 D$ ). We determined the levels of anti-SARS-CoV-2 antinucleocapsid protein $(\mathrm{N})$ antibodies in the serum of the patient, to assess her own antibody response to SARS-CoV-2, in the presence of circulating casirivimab and imdevimab. The patient had detectable anti-N antibodies in her serum on day 14, and their titers were found to have increased on days 21 and 28 (Fig. $1 E$ ).

We also collected longitudinal whole-blood samples from the patient at various time points from day 1 to day 14 . We performed whole-blood RNA sequencing (RNA-seq) with hemoglobin RNA depletion. We analyzed the transcripts of genes involved in antiviral immunity. We found that type I IFN transcripts, including that for IFN- $\beta$, were undetectable or only detectable in very low amounts at all time points, including days 1 to 4 , although IFN- $\alpha 2$ protein was detected in the serum by Simoa (Fig. $2 A$ ). The source of the IFN- $\alpha 2$ in the patient's blood may have been the upper respiratory tract (37). Furthermore, the frequency of plasmacytoid dendritic cells (pDCs) among leukocytes may be too low for the detection of their intrinsic type I IFN mRNA production by whole-blood RNAseq. Either way, the absence of detectable type I IFN transcripts in the patient's leukocytes is consistent with a lack of autoamplification of type I IFNs in the absence of IRF9 and ISGF-3. However, we found that the levels of transcripts of 140 previously defined type I IFN ISGs (5), 44 of which are known to be antiviral (38), were high before mAb therapy (Fig. 2). This is consistent with the initial sensing of viral infection by pDCs (39). These ISGs are probably triggered by the virus via IRF3, IRF5, and IRF7, but not IRF9 (which binds ISRE DNA motifs in ISGF-3 complexes). Moreover, no ISG induction was observed on days 3 and 4, despite a strong increase in serum IFN- $\alpha 2$ protein levels, consistent with the abolition of type I IFN signaling in the blood cells of the patient (Fig. 2). Thus, our findings indicate that SARS-CoV-2 induced ISRE-driven
ISGs in leukocytes via transcription factors other than the IRF9-containing ISGF-3-presumably therefore via IRF3, IRF5, and IRF7-whereas leukocytes displayed no type I IFN induction (other than possibly in $\mathrm{pDCs}$ ) and did not respond to type I IFNs made by other cells or tissues, such as pDCs and upper respiratory tract tissues. We further compared our patient's ISGs signature ( $z$-score) to that of other patients infected with SARS-CoV-2, hospitalized within the first week since symptom onset, and sampled longitudinally (5) (SI Appendix, Fig. S1). Patients whose medical state deteriorated and who succumbed all had a high ISG $z$-score early during COVID-19 or before death, whereas patients who survived had a lower ISG $z$-score along the course of recovery. Our patient's very high ISG $z$-score on day 1 therefore further suggested that she might have developed a life-threatening form of COVID19 had she not received the mAbs neutralizing SARS-CoV-2.

We, thus, report the safety and efficacy of combined casirivimab and imdevimab therapy for preventing the development of pneumonia and life-threatening COVID-19 in a child with AR complete IRF9 deficiency whose cells do not activate ISGF-3 or display induction of the ISGs activated by this transcription factor in response to both type I and III IFNs. The efficacy of antibody-mediated viral neutralization in the patient was demonstrated by the rapid resolution of her clinical manifestations. Further evidence of efficacy was provided by: 1) the rapid decrease in nasal viral load, as reported in other patients (25); and 2) the rapid clearance of SARS-CoV-2 viremia, which was initially predictive of a severe outcome (35). Patients with AR IRF9, STAT1, or STAT2 deficiencies, and disrupted cellular responses to both type I and III IFNs, and patients with IFNAR1 or IFNAR2 deficiencies may benefit from the same approach. It is unclear whether patients with AR interleukin$10 \mathrm{RB}$ deficiency, resulting in a lack of cell response to type III but not to type I IFNs, are at risk for severe COVID-19. Moreover, patients with auto-Abs neutralizing not only IFN- $\alpha$ and $-\omega$, but also IFN- $\beta$, might also benefit from this approach, whereas IFN- $\beta$ (alone or in combination with mAbs) may remain a treatment of choice in the absence of detectable IFN$\beta$-neutralizing auto-Abs $(23,40)$. This observation further suggests that children with inborn errors of, or auto-Abs to type I IFNs, should be vaccinated against SARS-CoV-2. This observation is also important for the general population, as it provides proof-of-concept that mAbs specific for SARS-CoV-2 can efficiently prevent critical pneumonia, even in the patients most vulnerable to SARS-CoV-2, with inheritably compromised type I and III IFN immunity.

\section{Material and Methods}

PCR on Nasal Swabs and Blood. PCR was performed on nasal swabs with the Alinity kit (Abbott). PCR was performed on blood with the SIMPLEXA COVID19 direct assay (DiaSorin).

Antigenemia. Serum $\mathrm{N}$ antigen concentrations were determined with the COV-QUANTO ELISA kit (AAZ)

IFN- $\alpha 2$ Determination by Simoa. Serum IFN- $\alpha 2$ concentrations were determined with Quanterix platforms, as previously described (36).

RNA-seq. We collected whole blood from the patient on days 1, 3, 4, and 14, with a PAXgene Blood RNA tube. The blood sample was subjected to hemoglobin RNA depletion. Samples were sequenced on the Illumina NextSeq platform with a single-end 75-bp configuration. The RNA-seq fastq raw data were inspected to ensure that they were of high quality. The sequencing reads were mapped onto the human reference genome GRCh38 with STAR aligner V2.7, and the mapped reads were then quantified to determine the gene-level read counts, with featureCounts v2.0.2. The gene-level read counts were normalized and $\log _{2}$-transformed with DESeq2, to obtain the gene expression profile for all samples. 
A

Type I IFNs ISGs with anti-viral functions Total ISGs Protein-coding genes
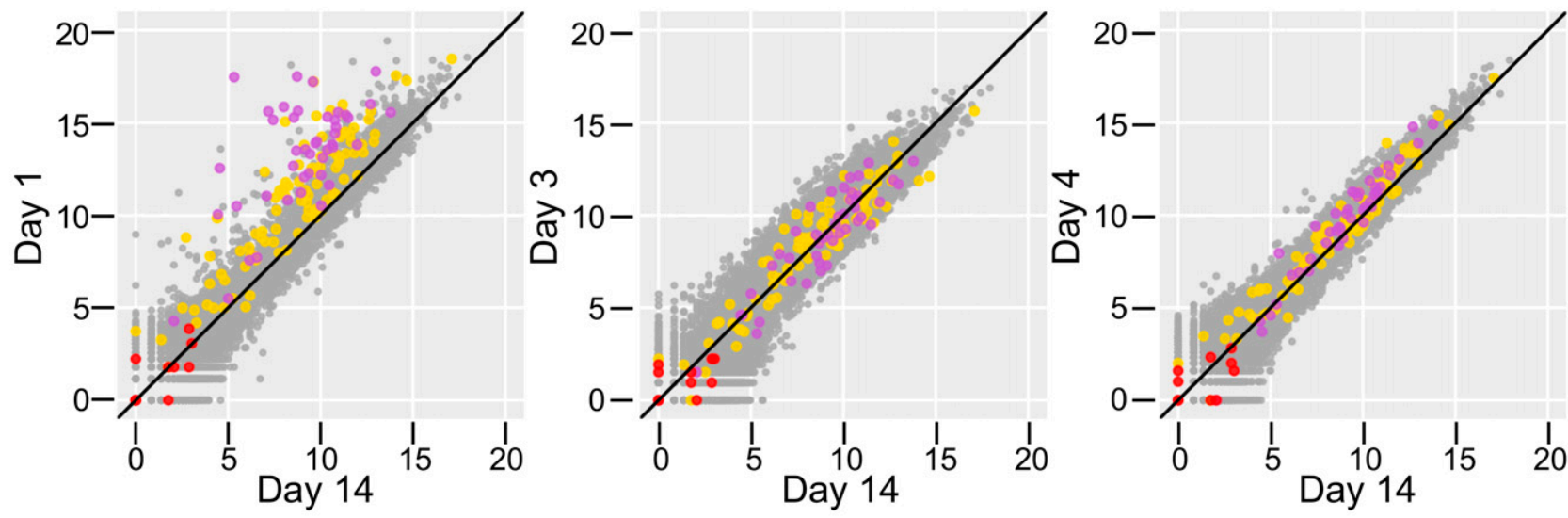

B

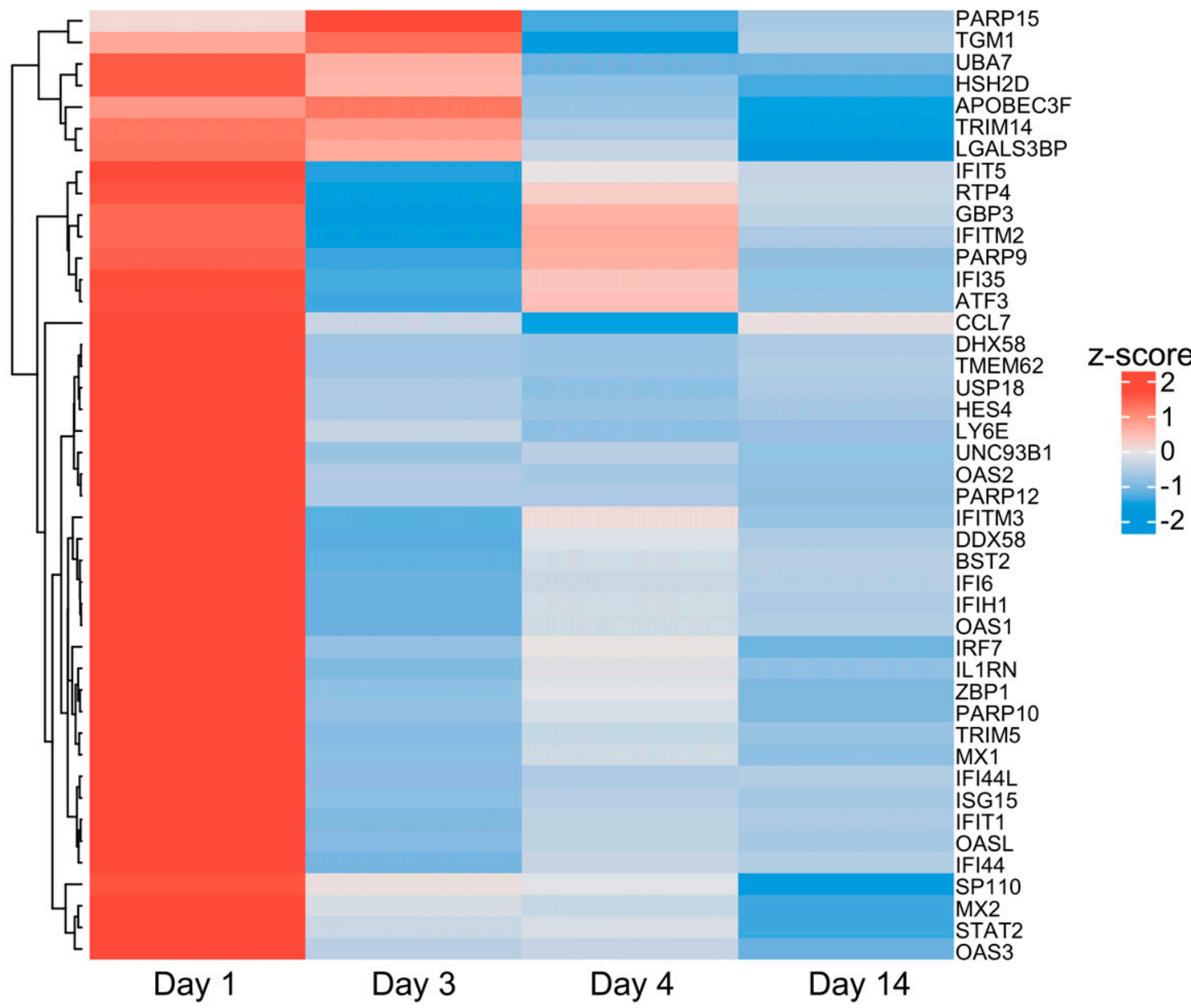

Fig. 2. (A) RNA-seq comparison of gene expression between day 1, day 3, day 4, and day 14 in the patient, with expression assessed as the normalized and log-transformed read count (red: type-I IFN genes; purple: ISGs known to have antiviral functions; yellow: other ISGs; gray: other protein-coding genes). (B) Heatmap of RNA-seq gene expression $z$-scores for 44 ISGs with known antiviral functions in the patient on day 1 , day 3 , day 4, and day 14.

Patient Recruitment and Ethics. Clinical history and biological specimens were obtained from the referring clinicians, upon verification that a signed consent was available for each participant in the study. All the experiments involving human subjects conducted in this study were performed in accordance with institutional, local, and national ethical guidelines. Approval was obtained from the French Ethics Committee "Comité de Protection des Personnes," the French National Agency for Medicine and Health Product Safety, the "Institut
National de la Santé et de la Recherche Médicale," in France (protocol no. C10-13, ID-RCB no. 2010-A00634-35), and the Rockefeller University Institutional Review Board in New York (protocol no. JCA-0700).

Data Availability. All study data are included in the article and SI Appendix. The whole blood RNA-sequencing data are available in NCBI-SRA database, under project ID PRJNA770903. 
ACKNOWLEDGMENTS. We thank Ivona Aksentijevich for critical reading of this manuscript. The Laboratory of Human Genetics of Infectious Diseases is supported by the HHMI, the Rockefeller University; the St. Giles Foundation; the NIH (R01AI088364); the National Center for Advancing Translational Sciences; NIH Clinical and Translational Science Award program (UL1TR001866); a Fast Grant from Emergent Ventures; Mercatus Center at George Mason University, the Yale Center for Mendelian Genomics and the Genome Sequencing Program Coordinating Center funded by the National Human Genome Research Institute (UM1HG006504 and U24HG008956); the Fisher Center for Alzheimer's Research Foundation; the Meyer Foundation; the French National Research Agency (ANR) under the "Investments for the Future" program (ANR-10-IAHU-01); the Integrative Biology of Emerging Infectious Diseases

1. P. Brodin, Immune determinants of COVID-19 disease presentation and severity. Nat Med. 27, 28-33 (2021).

2. Q. Zhang et al., COVID-STORM Clinicians; COVID Clinicians; Imagine COVID Group; French COVID Cohort Study Group; CoV-Contact Cohort; Amsterdam UMC Covid-19 Biobank; COVID Human Genetic Effort; NIAID-USUHS/TAGC COVID Immunity Group, Inborn errors of type I IFN immunity in patients with life-threatening COVID-19. Science 370, eabd4570 (2020).

3. T. Asano et al., COVID Human Genetic Effort; COVID-STORM Clinicians; COVID Clinicians; Imagine COVID Group; French COVID Cohort Study Group; CoV-Contac Cohort; Amsterdam UMC Covid-; Biobank; NIAID-USUHS COVID Study Group, Xlinked recessive TLR7 deficiency in $\sim 1 \%$ of men under 60 years old with lifethreatening COVID-19. Sci. Immunol. 6, eabl4348 (2021).

4. P. Bastard et al., HGID Lab; NIAID-USUHS Immune Response to COVID Group; COVID Clinicians; COVID-STORM Clinicians; Imagine COVID Group; French COVID Cohort Study Group; Milieu Intérieur Consortium; CoV-Contact Cohort; Amsterdam UMC Covid-19 Biobank; COVID Human Genetic Effort, Autoantibodies against type I IFNs in patients with life-threatening COVID-19. Science 370, eabd4585 (2020).

5. M. G. P. van der Wijst et al., UCSF COMET Consortium, Type I interferon autoantibodies are associated with systemic immune alterations in patients with COVID-19. Sci. Transl. Med. 13, eabh2624 (2021)

6. P. Bastard et al., HGID Lab; COVID Clinicians; COVID-STORM Clinicians; NIAID Immune Response to COVID Group; NH-COVAIR Study Group; Danish CHGE; Danish Blood Donor Study; St. James's Hospital; SARS CoV2 Interest group; French COVID Cohor Study Group; Imagine COVID-Group; Milieu Intérieur Consortium; CoV-Contact Cohort; Amsterdam UMC Covid-19; Biobank Investigators; COVID Human Genetic Effort; CONSTANCES cohort; 3C-Dijon Study; Cerba Health-Care; Etablissement du Sang study group, Autoantibodies neutralizing type I IFNs are present in $\sim 4 \%$ of uninfected individuals over 70 years old and account for $\sim 20 \%$ of COVID-19 deaths. Sci. Immunol. 6, eabl4340 (2021).

7. R. Koning, P. Bastard, J. L. Casanova, M. C. Brouwer, D. van de Beek; with the Amster dam U.M.C. COVID-19 Biobank Investigators, Autoantibodies against type I interferons are associated with multi-organ failure in COVID-19 patients. Intensive Care Med. 47, 704-706 (2021)

8. E. Y. Wang et al., Diverse functional autoantibodies in patients with COVID-19. Nature 595, 283-288 (2021).

9. S. E. Vazquez et al., Neutralizing autoantibodies to type I interferons in COVID-19 convalescent donor plasma. J. Clin. Immunol. 41, 1169-1171 (2021).

10. C. G. K. Ziegler et al., Impaired local intrinsic immunity to SARS-CoV-2 infection in severe COVID-19. Cel/ 184, 4713-4733.e22 (2021)

11. X. Solanich et al., Pre-existing Autoantibodies Neutralizing High Concentrations of Type I Interferons in Almost 10\% of COVID-19 Patients Admitted to Intensive Care in Barcelona. J. Clin. Immunol. (2021).

12. Y. Acosta-Ampudia et al., COVID-19 convalescent plasma composition and immunological effects in severe patients. J. Autoimmunity 118, 102598 (2021)

13. A. Chauvineau-Grenier et al., Autoantibodies neutralizing type I interferons in $20 \%$ of COVID-19 deaths in a French hospital. Res. Sq. (2021).

14. J. Troya et al., Neutralizing autoantibodies to type I IFNs in $>10 \%$ of patients with severe COVID-19 pneumonia hospitalized in Madrid, Spain. J. Clin. Immunol. 41, 914-922 (2021)

15. D. Goncalves et al., Antibodies against type I interferon: Detection and association with severe clinical outcome in COVID-19 patients. Clin. TransI. Immunology 10, e1327 (2021).

16. M. S. Abers et al., Neutralizing type-l interferon autoantibodies are associated with delayed viral clearance and intensive care unit admission in patients with COVID-19. Immunol. Cell Biol. 99, 917-921 (2021).
Laboratory of Excellence (ANR-10-LABX-62-IBEID); the French Foundation for Medical Research (FRM) (EQU201903007798); the FRM and ANR GENCOVID project; the ANRS-COV05, ANR GENVIR (ANR-20-CE93-003), ANR AABIFNCOV (ANR-20-CO11-0001), and ANR GenMIS-C (ANR-21-COVR0039) projects; the European Union's Horizon 2020 research and innovation program under Grant 824110 (EASI-genomics); the Square Foundation; Grandir-Fonds de solidarité pour l'enfance; the SCOR Corporate Foundation for Science; Fondation du Souffle; INSERM; and the University of Paris. R.L. was supported by the Fondation Bettencourt-Schueller (INSERM-Bettencourt program). P.B. was supported by the MD-PhD program of the Imagine Institute (with the support of the Fondation Bettencourt-Schueller)

17. I. Meyts, J.-L. Casanova, Viral infections in humans and mice with genetic deficiencies of the type I IFN response pathway. Eur. J. Immunol. 51, 1039-1061 (2021)

18. H. K. Lim et al., Severe influenza pneumonitis in children with inherited TLR3 deficiency. J. Exp. Med. 216, 2038-2056 (2019).

19. M. J. Ciancanelli et al., Infectious disease. Life-threatening influenza and impaired interferon amplification in human IRF7 deficiency. Science 348, 448-453 (2015).

20. J.-L. Casanova, H. C. Su; COVID Human Genetic Effort, A global effort to define the human genetics of protective immunity to SARS-CoV-2 infection. Cell 181, 1194-1199 (2020).

21. Q. Zhang, et al., COVID Human Genetic Effort, Life-threatening COVID-19: Defective interferons unleash excessive inflammation. Med. (N.Y.) 1, 14-20 (2020).

22. R. Lévy et al., Correction to: IFN- $\alpha 2$ a therapy in two patients with inborn errors of TLR3 and IRF3 infected with SARS-CoV-2. J. Clin. Immunol. 41, 28 (2021).

23. P. Bastard et al., Interferon- $\beta$ therapy in a patient with incontinentia pigmenti and autoantibodies against type I IFNs infected with SARS-CoV-2. J. Clin. Immunol. 41 931-933 (2021).

24. D. C. Vinh et al., COVID Human Genetic Effort, Harnessing type I IFN immunity against SARS-CoV-2 with early administration of IFN- $\beta$. J. Clin. Immunol. 41, 1425-1442 (2021).

25. D. M. Weinreich et al., Trial Investigators, REGN-COV2, a neutralizing antibody cocktail, in outpatients with Covid-19. N. Engl. J. Med. 384, 238-251 (2021).

26. J. Wolf et al., Initial guidance on use of monoclonal antibody therapy for treatment of coronavirus disease 2019 in children and adolescents. J. Pediatric Infect. Dis. Soc 10, 629-634 (2021)

27. E. M. N. Ferré et al., SARS-CoV-2 spike protein-directed monoclonal antibodies may ameliorate COVID-19 complications in APECED patients. Front. Immunol. 12, 720205 (2021).

28. N. Hernandez et al., Life-threatening influenza pneumonitis in a child with inherited IRF9 deficiency. J. Exp. Med. 215, 2567-2585 (2018).

29. H. M. Lazear, J. W. Schoggins, M. S. Diamond, Shared and distinct functions of type I and type III interferons. Immunity 50, 907-923 (2019).

30. E. Platanitis et al., A molecular switch from STAT2-IRF9 to ISGF3 underlies interferoninduced gene transcription. Nat. Commun. 10, 2921 (2019).

31. G. R. Stark, J. E. Darnell, Jr, The JAK-STAT pathway at twenty. Immunity 36, 503-514 (2012).

32. F. Konings et al., SARS-CoV-2 variants of interest and concern naming scheme conducive for global discourse. Nat. Microbiol. 6, 821-823 (2021).

33. J. Hansen et al., Studies in humanized mice and convalescent humans yield a SARSCoV-2 antibody cocktail. Science 369, 1010-1014 (2020).

34. A. C. Hurt, A. K. Wheatley, Neutralizing antibody therapeutics for COVID-19. Viruses 13, 628 (2021).

35. Y. Li et al., SARS-CoV-2 viremia is associated with distinct proteomic pathways and predicts COVID-19 outcomes. J. Clin. Invest. 131, 148635 (2021).

36. K. Dorgham et al., Distinct cytokine profiles associated with COVID-19 severity and mortality. J. Allergy Clin. Immunol. 147, 2098-2107 (2021).

37. A. R. Daamen et al., Comprehensive transcriptomic analysis of COVID-19 blood, lung and airway. Sci. Rep. 11, 7052 (2021).

38. W. M. Schneider, M. D. Chevillotte, C. M. Rice, Interferon-stimulated genes: A complex web of host defenses. Annu. Rev. Immunol. 32, 513-545 (2014).

39. F. Onodi et al., SARS-CoV-2 induces human plasmacytoid predendritic cell diversification via UNC93B and IRAK4. J. Exp. Med. 218, e20201387 (2021).

40. P. D. Monk et al., Inhaled Interferon Beta COVID-19 Study Group, Safety and efficacy of inhaled nebulised interferon beta-1a (SNG001) for treatment of SARS-CoV-2 infec tion: A randomised, double-blind, placebo-controlled, phase 2 trial. Lancet Respir. Med. 9, 196-206 (2021) 